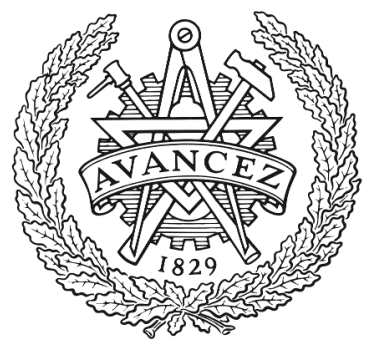

CHALMERS

UNIVERSITY OF TECHNOLOGY

\title{
Surface modification of polytetrafluoroethylene thin films by non-coherent UV light and water treatment for electrowetting applications
}

Downloaded from: https://research.chalmers.se, 2023-04-26 02:07 UTC

Citation for the original published paper (version of record):

López, C., Cedeño-Mata, M., Dominguez-Pumara, M. et al (2020). Surface modification of polytetrafluoroethylene thin films by non-coherent UV light and water treatment for electrowetting applications. Progress in Organic Coatings, 149.

http://dx.doi.org/10.1016/j.porgcoat.2020.105593

N.B. When citing this work, cite the original published paper. 


\title{
Surface modification of polytetrafluoroethylene thin films by non-coherent UV light and water treatment for electrowetting applications
}

\author{
Cristian Daniel López ${ }^{\mathrm{a}}$, Michelle Cedeño-Mata ${ }^{\mathrm{b}}$, Manuel Dominguez-Pumar ${ }^{\mathrm{b}}$, Sandra Bermejo ${ }^{\mathrm{b}, *}$ \\ ${ }^{a}$ Department of Space, Earth and Environment, Onsala Space Observatory, Group for Advanced Receiver Development (GARD), Chalmers University, Kemivägen 9, Plan 6, \\ Gard Group (MC2), PC 412 96, Gotemburg, Sweden \\ ${ }^{\mathrm{b}}$ Polytechnic University of Catalonia (UPC), Electronic Engineering Department, MNT Group, C/Jordi Girona 1-3, 0834, Barcelona, Spain
}

\section{A B S T R A C T}

Hypothesis: The electrowetting on dielectric or EWOD phenomenon is used in a

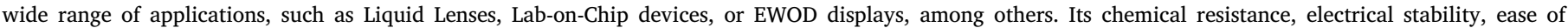

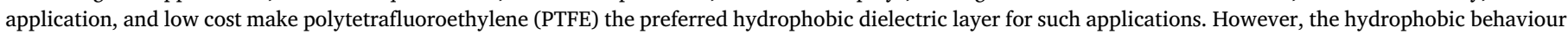

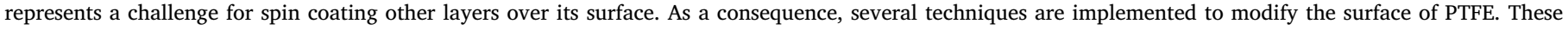

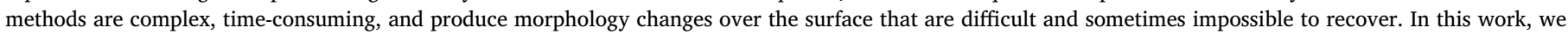

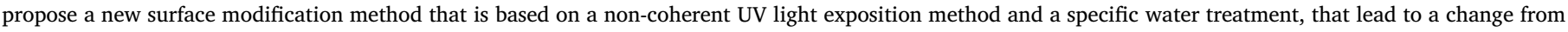
hydrophobic to hydrophilic, and a perfect recovery from hydrophilic to hydrophobic behaviour.

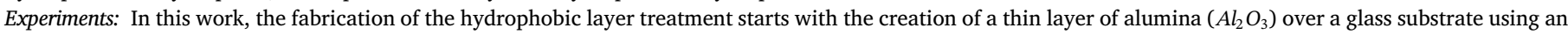

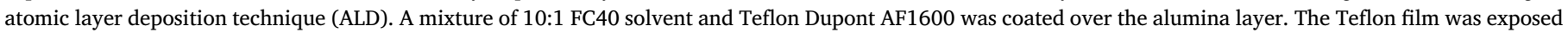

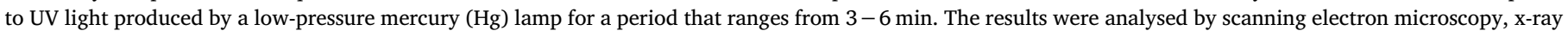
spectroscopy, and static deionized water contact angle measurements.

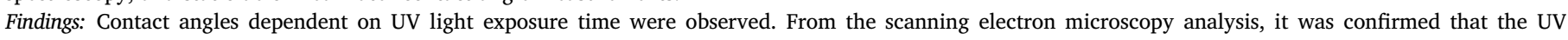

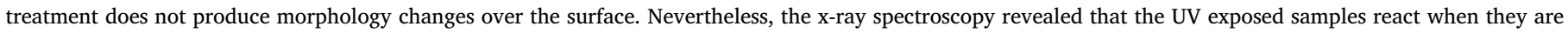

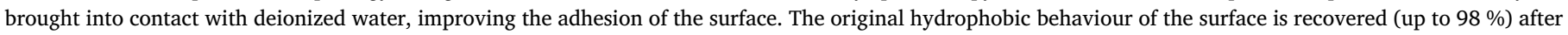
$3 \mathrm{~h}$ of thermal treatment. Furthermore, the thermal recovery analysis reveals a correlation between the recovery percentage and the applied temperature.

\section{Introduction}

Among the wide range of superhydrophobic and hydrophobic coatings [1,2], polytetrafluoroethylene (PTFE) is widely used for electrowetting on dielectric applications were a low-cost hydrophobic layer is needed. Nevertheless, this material faces important challenges. One of these may be the porosity of the layer that can eventually lead to electrical breakdown. This problem can be partially solved using a twolayer dielectric approach, which combines a few nanometers of alumina layer with a micron layer of PTFE. Another challenge may be the hydrophobic characteristic of the surface since there is always the need to spin a resin layer onto the PTFE layer. Consequently, to increase the adhesion of the surface, several different treatments have been proposed in the literature: chemical etching with sodium naphthalenide $\left(\mathrm{C}_{10} \mathrm{H}_{8} \mathrm{Na}\right)$, oxygen plasma etching, argon plasma etching, argon ion beam etching [3-5], dielectric barrier discharge plasma [6], excimer UV radiation [7] and $\gamma$-ray irradiation [8]. In the past several decades, chemical etching of PTFE surfaces using sodium naphthalene solutions has been widely adopted in the industry [3]. In order to generate changes in the surface wettability, the PTFE films are immerse in solutions rich in sodium naphthalene for $30-60 \mathrm{~s}$ at $50{ }^{\circ} \mathrm{C}$. Although this particular surface modification method proves to be simple and fast, it involves the use of harmful chemical compounds. Moreover, the surface morphology after such treatments reveal the presence of widespread cracks and defects that prevent wettability recovery. An alternative approach to enhance the adhesion of fluoropolymer surfaces is plasma treatment [3-5]. A variety of morphological and chemical modifications take place over the polymer surface when is exposed to plasma gases. Among the surface modification mechanisms, physical bombardment by energetic ions, chemical reactions at the surface and crosslinking are the most relevant. Short periods of tens of a second suffice to produce changes in the films. Nevertheless, the method requires a complex and costly setup. Additionally, morphological changes over the surface are hardly reversible. Ion beam treatment has been reported as a fast and effective solution to improve PTFE adhesion [3]. It consists in bombarding the sample with ions of argon and oxygen to

\footnotetext{
* Corresponding author.

E-mail address: sandra.bermejo@upc.edu (S. Bermejo).
} 
Table 1

Recipe for $1 \mu \mathrm{m}$ Teflon layer.

\begin{tabular}{lll}
\hline Step & Parameter & Value \\
\hline 1 & Velocity & $500 \mathrm{rpm} / \mathrm{s}$ \\
& Acceleration & $300 \mathrm{rpm}$ \\
& Time & $10 \mathrm{~s}$ \\
2 & Velocity & $3000 \mathrm{rpm}$ \\
& Acceleration & $300 \mathrm{rpm} / \mathrm{s}$ \\
& Time & $60 \mathrm{~s}$
\end{tabular}

Table 2

UV Contact angle for different exposure times.

\begin{tabular}{llllll}
\hline Exposure Time (minutes) & 0 & 3 & 4 & 5 & 6 \\
\hline Contact Angle $\left({ }^{\circ}\right)$ & 123 & 94 & 72 & 51 & 31 \\
\hline
\end{tabular}

produce morphological and chemical changes. However, analogously to plasma treatments, the morphology changes avoid the recovering of the initial wettability. In [6], the effects of DBD or Dielectric Barrier Discharge Plasma over PTFE were investigated. A Dielectric Barrier Discharge Plasma system is composed of 2 electrodes spaced by few millimeters where air plasma at atmospheric pressure is generated. The samples are bound to the lower electrode and are displaced-mechanically back and forth to avoid non-uniformities in the treatment. The morphology changes associated with this technique are comparatively smaller than in plasma-etched surfaces. Nonetheless, it remains to be seen if the surface can be properly recovered through thermal annealing. In [7], excimer UV radiation has been extensively investigated as a surface modification technique for polymer films. The irradiation of PTFE samples with excimer laser pulses induce etching over the surface and alter the surface morphology. As a consequence, the wettability changes dramatically. Cracks, bubbles and disruptions are frequently observed in the treated sample, which clearly compromises the possibility of surface recovery. As it was investigated in [8], $\gamma$-ray radiation could potentially be used for surface modification of PTFE. However, the highly specialized setup needed to perform the treatment makes this method hard to implement. These treatments have different advantages and disadvantages, but all of them produce morphology changes, which are difficult to recover and, in some cases, produce irreversible effects. Since it is desired to fully recover the hydrophobic behaviour after resin development, the commonly used methods are not always suitable for electrowetting applications.

It is well known that UV light can be used for surface activation purposes [9,7,10-14]. In this process, the high energy UV photons break up chemical bonds at the surface. This bond forms new compounds depending on the gases or liquids that are present during and after the treatment. Since it is often performed at atmospheric pressure, oxygen and its photooxidation derivate, as ozone, play a major role in the improvement of wettability due to the increment of surface energy purposes $[9,15]$. In spite of its simplicity and scalability, this treatment has not been reported yet for EWOD applications. In this paper, this method is performed, characterized and analysed.

\section{Experimental}

\subsection{Sample fabrication}

The fabrication process starts with the deposition of a $25 \mathrm{~nm}$ alumina layer over glass substrates using an atomic layer deposition technique. A layer of hexamethyldisilazane (HMDS) is then deposited onto the alumina layer. Our previous work [16] has proved that the use of HMDS not only increases the adhesion over alumina but also gets better contact angle values over the hydrophobic layer. A mixture of 10:1 FC40 solvent and Teflon Dupont AF1600 was prepared and

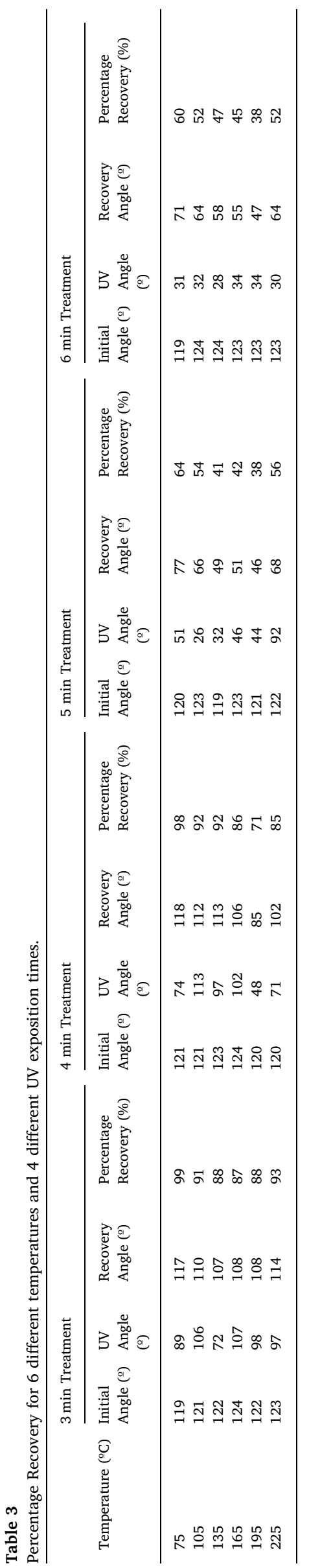




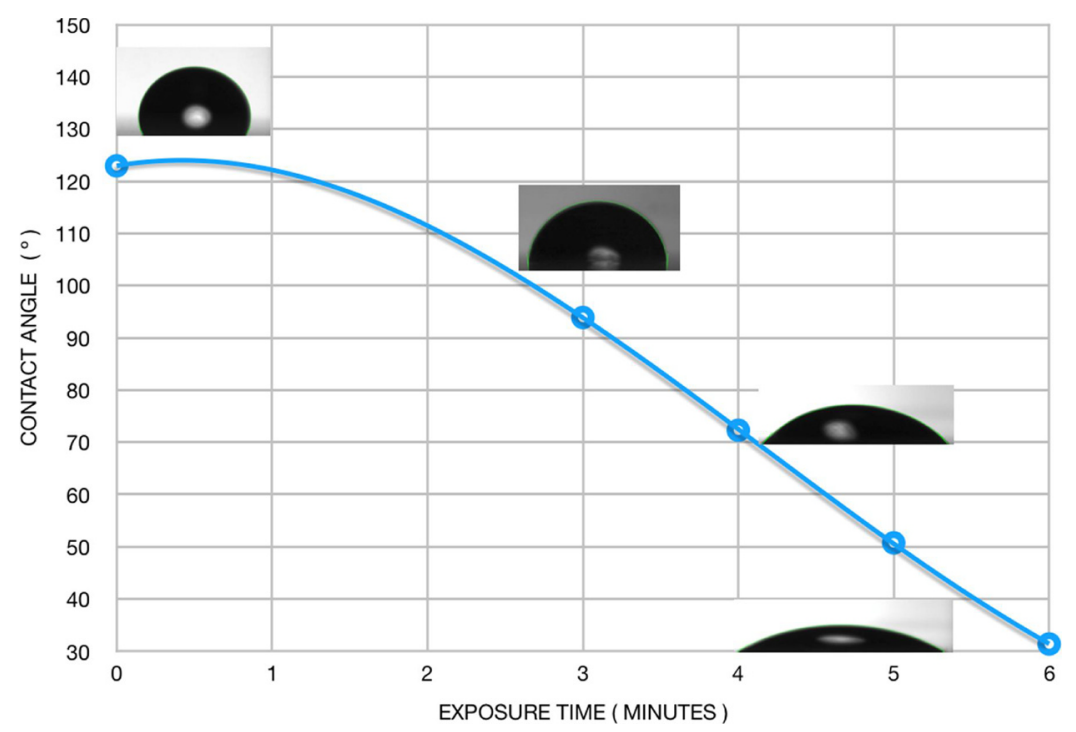

Fig. 1. Relation between contact angle and exposure time.

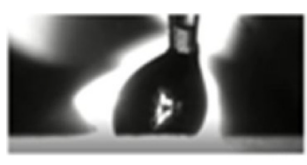

(a)

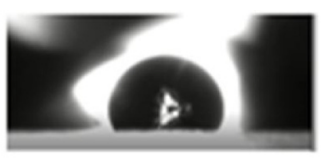

(b)

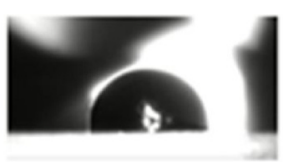

(c)

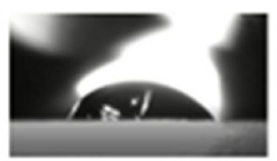

(d)

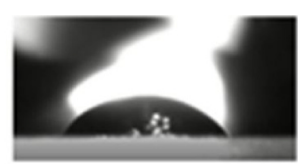

(e)

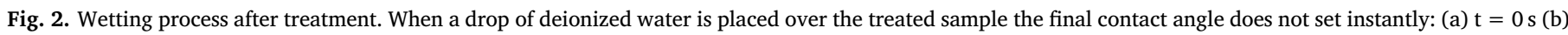
$\mathrm{t}=2 \mathrm{~s}(\mathrm{c}) \mathrm{t}=11 \mathrm{~s}(\mathrm{~d}) \mathrm{t}=44 \mathrm{~s}(\mathrm{e}) \mathrm{t}=1.36 \mathrm{~min}$.

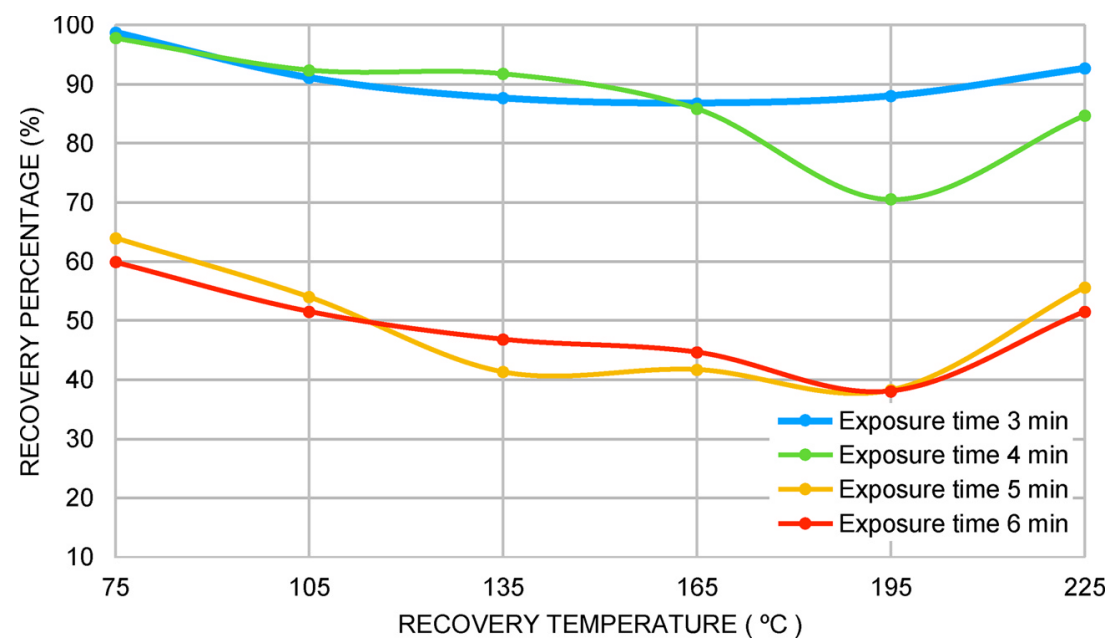

Fig. 3. Relation between Recovery percentage and Recovery time.

spinned-coated over this layer according to the recipe shown in Table 1. After that, the samples were heated in an oven at $105^{\circ} \mathrm{C}$ for $10 \mathrm{~min}$ and then put in a hot plate at $165^{\circ} \mathrm{C}$ for $5 \mathrm{~min}$. The PTFE layer has a thickness of $1 \mu \mathrm{m}$.

\subsection{UV treatment}

The UV treatment was performed inside a closed stainless steel compartment without vacuum. The distance between the low-pressure mercury lamp and the sample surface was $5 \mathrm{~mm}$. The average irradiance of the lamp was $30 \mathrm{~mW} / \mathrm{cm}^{2} @ 253 \mathrm{~nm}$ wavelength. The samples were left inside the chamber for periods of 3,4,5 and $6 \mathrm{~min}$.

\subsection{Contact angle measurement}

The static contact angle was measured for 24 samples in three different time steps: before and after the UV treatment, and after the thermal recovery. The initial contact angle was obtained from the average of the 24 samples. After that, in order to measure the effect of the 4 different applied exposure times, the 24 samples were separated into 4 groups of 6 samples. In each case $10 \mu \mathrm{L}$ drops of deionized water were carefully placed over the surface and the contact angle was obtained through image processing. Left and right contact angles were measured separately. Regarding surface recovery, the hydrophobic behaviour of the samples was recovered for $3 \mathrm{~h}$ to 6 different temperatures, $75^{\circ} \mathrm{C}, 105^{\circ} \mathrm{C}, 135^{\circ} \mathrm{C}, 165^{\circ} \mathrm{C}, 195^{\circ} \mathrm{C}$, and $225^{\circ} \mathrm{C}$. The 


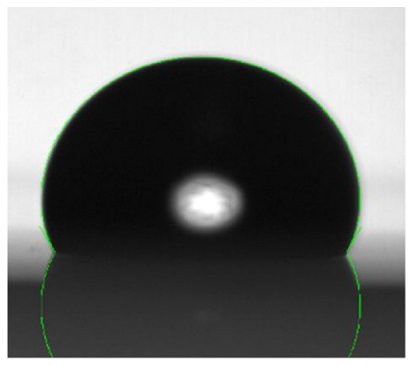

(a)

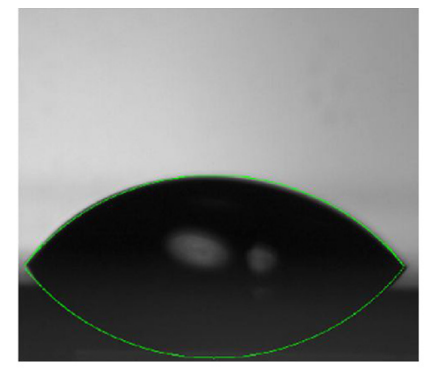

(b)

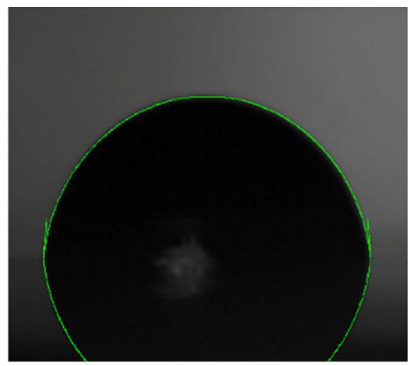

(c)

Fig. 4. Final angle contact: (a) Not treated sample, (b) Treated sample (UV exposition time of 4 min), and (c) Treated sample (Heat treatment $3 \mathrm{~h}$ at $165^{\circ} \mathrm{C}$ ).

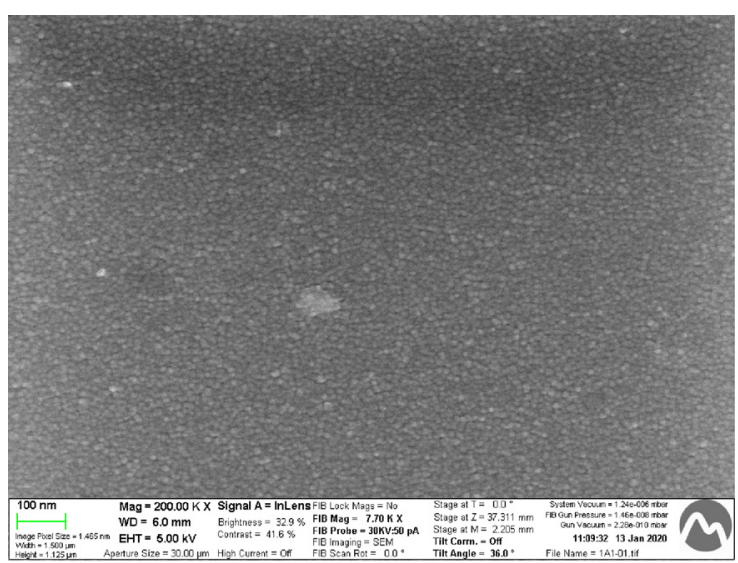

(a)

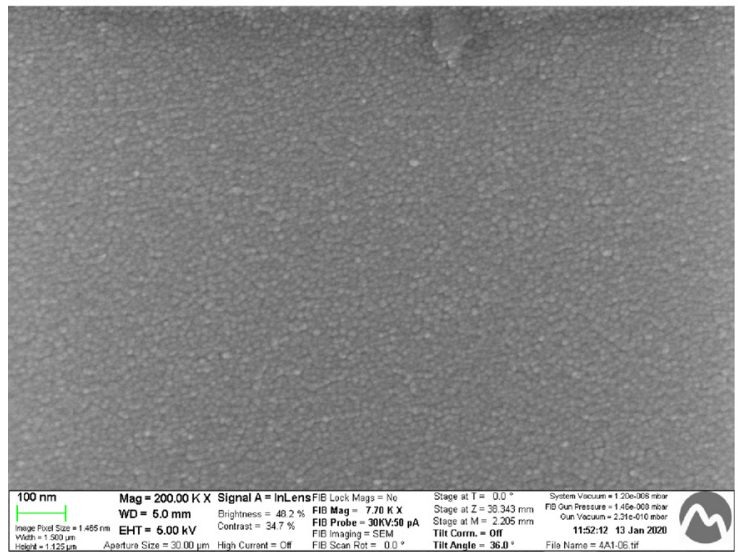

(c)

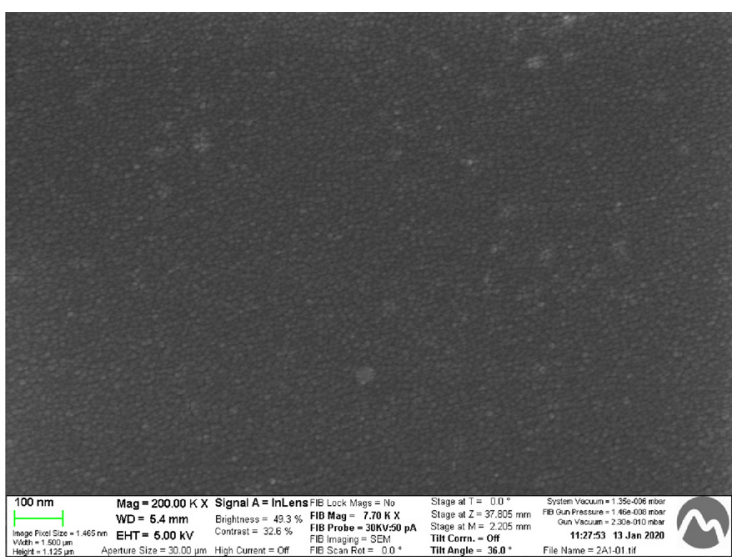

(b)

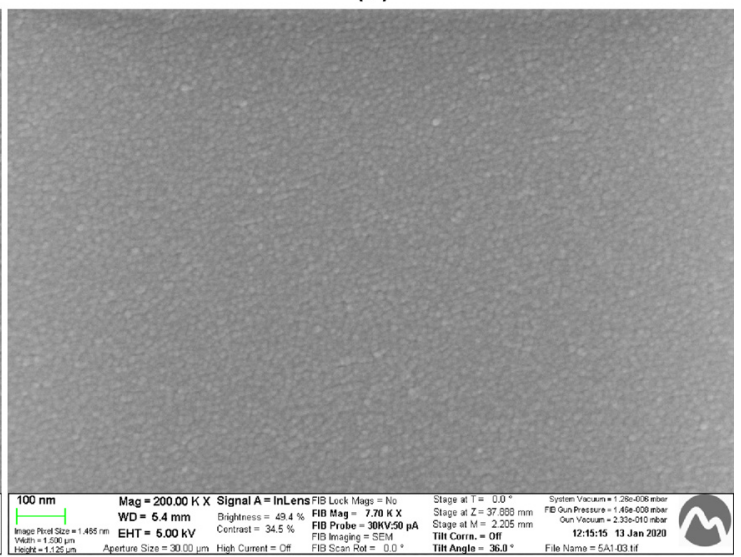

(d)

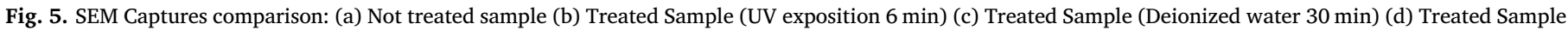
(Heat treatment $3 \mathrm{~h}$ ).

maximum employed temperature value was chosen based on the maximum temperature that the AF1600 can withstand.

\subsection{Morphological and chemical characterization}

Scanning electron microscopy (SEM) was used to analyse the surface morphology of the samples before and after the exposition to the UV light treatment. Besides, X-ray spectroscopy allowed the determination of the surface chemical composition before and after the exposition. The following compounds were tested: C 1s, O 1s, F 1s, Al 2p, and Si 2p. Carbon and Fluor are atoms that form the monomer of PTFE (CF2), while the oxygen content seems to be related to the wetting modification. Additionally, aluminum content was analysed to assess the porosity of the thin film. Silicon determination was performed since
Silicon is part of the chemical structure of HMDS (Table 2).

\section{Results and discussion}

\subsection{UV exposition time and contact angle correlation}

The contact angles averages are depicted in Table 3 for different exposure times. In Fig. 1 it is clearly seen that the contact angle diminishes as the exposure time increments. It is important to remark that the contact angle of a drop of water in contact with the modified surface takes up to $1.36 \mathrm{~min}$ to reach its final value. In other words, the wetting process occurs when deionized water is put in contact with the surface. This phenomenon was captured in a video, five captions of it are shown in Fig. 2. It could indicate that water itself plays a major role in the 


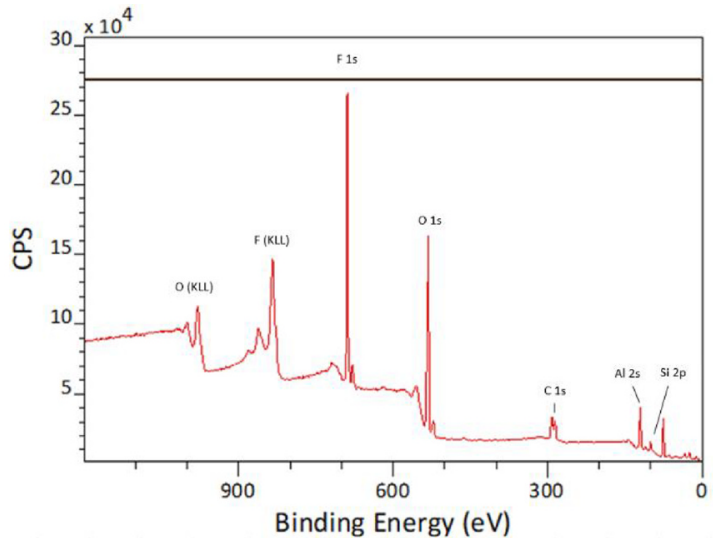

(a)

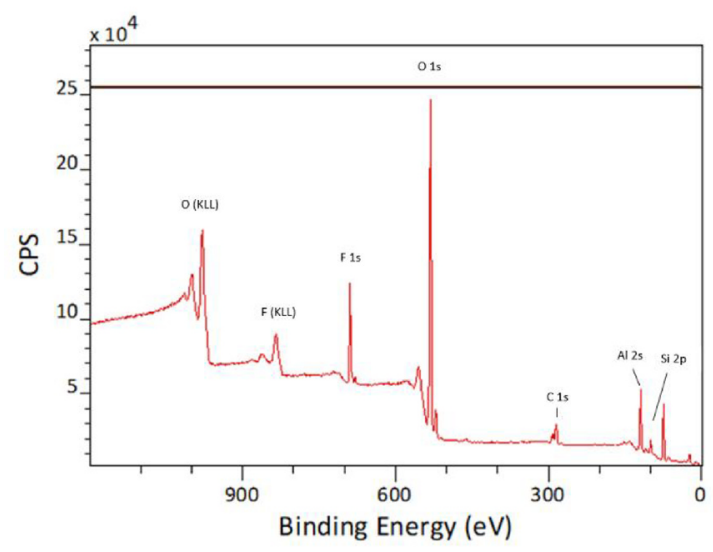

(c)

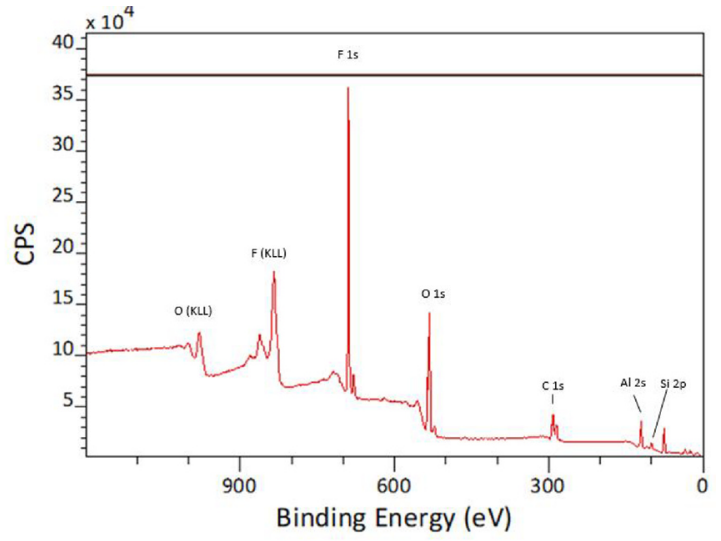

(b)

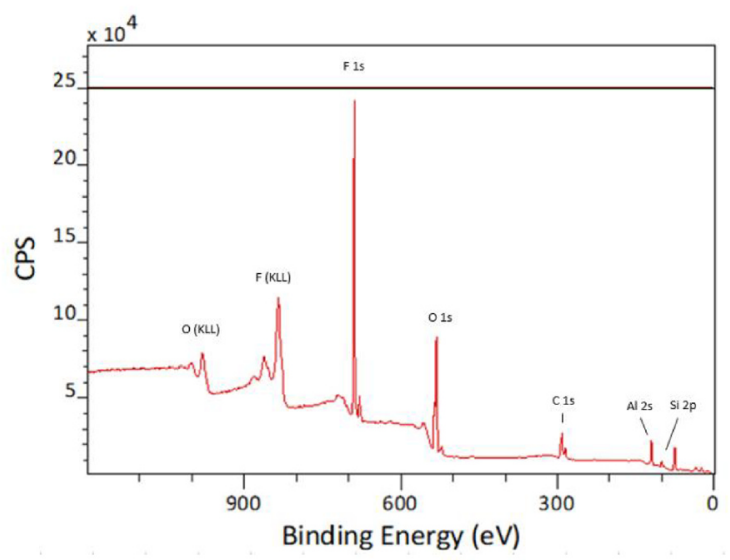

(d)

Fig. 6. XPS spectrums: (a) Reference Sample (b) Treated Sample (UV exposition $6 \mathrm{~min}$ ) (c) Treated Sample (UV exposition $6 \mathrm{~min}+30 \mathrm{~min}$ deionized water) (d) Sample Recovering $\left(3 \mathrm{~h}\right.$ at $\left.165^{\circ} \mathrm{C}\right)$.

process and that the main changes are chemical and not morphological. The variability in the contact angle value can be explained by the natural heterogeneity of PTFE (i.e. coexistence of crystalline and amorphous phases) that define different light absorption behaviour over the same sample.

\subsection{Thermal recovery}

The surface hydrophobicity can be recovered by thermal treatment. Table 3 depicts the results of samples treated at 6 different temperatures and 4 different exposition times. The recovery percentage is calculated as follows:

Recovery percentage $=\frac{\text { RecoveryAngle. } 100}{\text { InitialAngle }}$

The recovery percentage covers the range from $38 \%$ to the $98 \%$. The data show a correlation between the applied recovery temperatures, the employed exposure time, and the recovery percentage.

In particular, Fig. 3 shows the relationship between the applied temperature in the thermal recovery process and the achieved recovery percentage for the different applied exposure times. As depicted in Fig. 3, 6 different temperatures have been employed to recover the original contact angle. It is important to notice that the maximum applied temperature has been selected according to the maximum working temperature of the AF1600. The obtained curves reflect that the recovery percentage decreases when the applied exposure time increases. These variations between the final contact angle values and original ones could be due to the fact that increasing exposure time results in a larger drop-off in the contact angle, which in turn is more difficult to recover with the same applied heating treatment time. Furthermore, it is important to highlight that the recovery percentage increases when the applied recovery temperature decreases.

Fig. 4 displays the contact angle variation between hydrophobic and hydrophilic behaviour. In particular, Fig. 4a shows a contact angle of $120^{\circ}$ of a reference sample, Fig. $4 \mathrm{~b}$ illustrates a contact angle of $47^{\circ}$ result of a $4 \mathrm{~min}$ UV treated, while, Fig. $4 \mathrm{c}$ depicts a sample with a contact angle of $84^{\circ}$, which has been subjected to the entire process at $165^{\circ} \mathrm{C}$.

\subsection{Morphology changes}

In order to reveal the possible morphological changes due to the treatment, four samples were analysed using SEM. In Figs. 5a the reference sample is shown, in Fig. 5b a 6 min UV treated sample is illustrated, while, in $5 \mathrm{c}$ a UV and deionized water treated sample is displayed, and finally, in $5 \mathrm{~d}$ a sample which, has been subjected to the entire process. In all cases, a homogeneous nano-porous structure is seen. This is clearly different from other structures PTFE layers reported in the literature where the morphology of the layer is highly modified after different specific treatments $[3,6,7,16,17]$. Fig. 5 shows that there are not any visible cracks, spires, spherulites or morphological changes after the treatment. As a consequence, it can be concluded that the process does not morphologically damage the surface. 


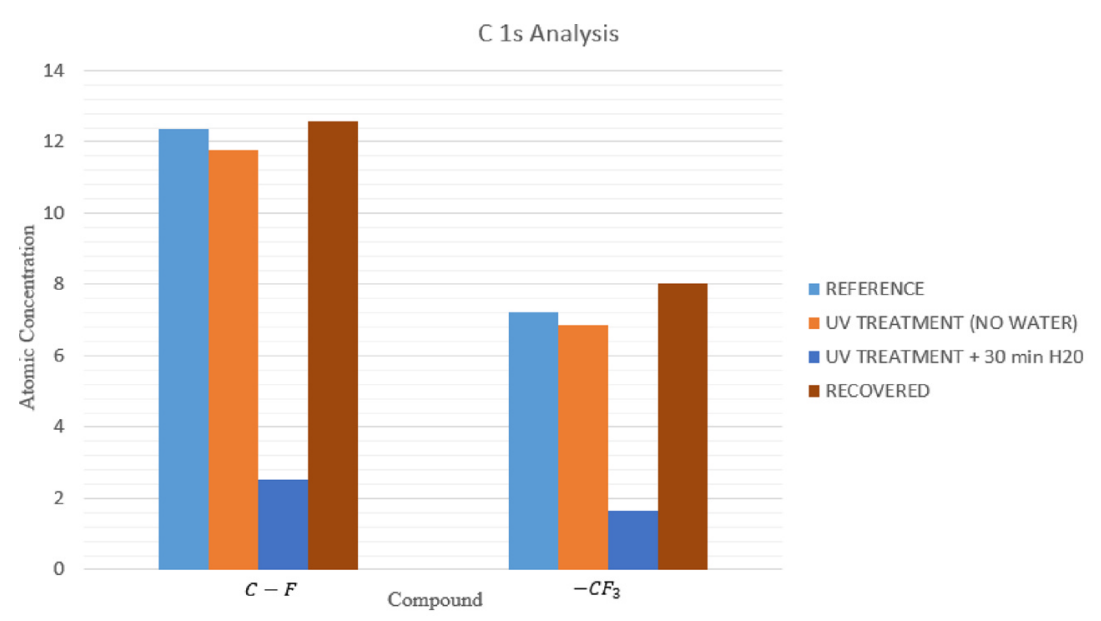

(a)

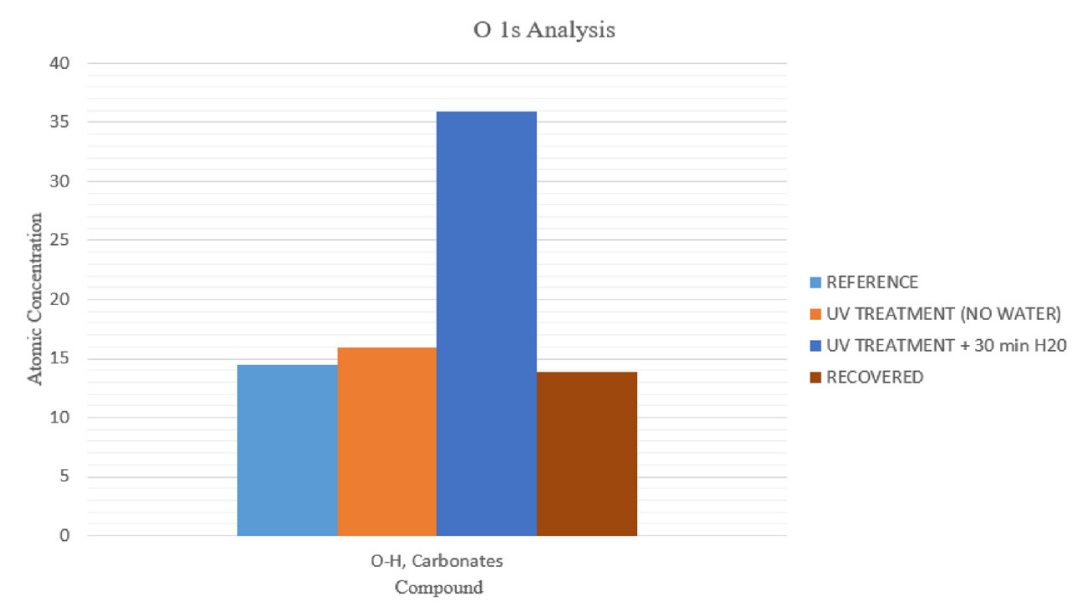

(b)

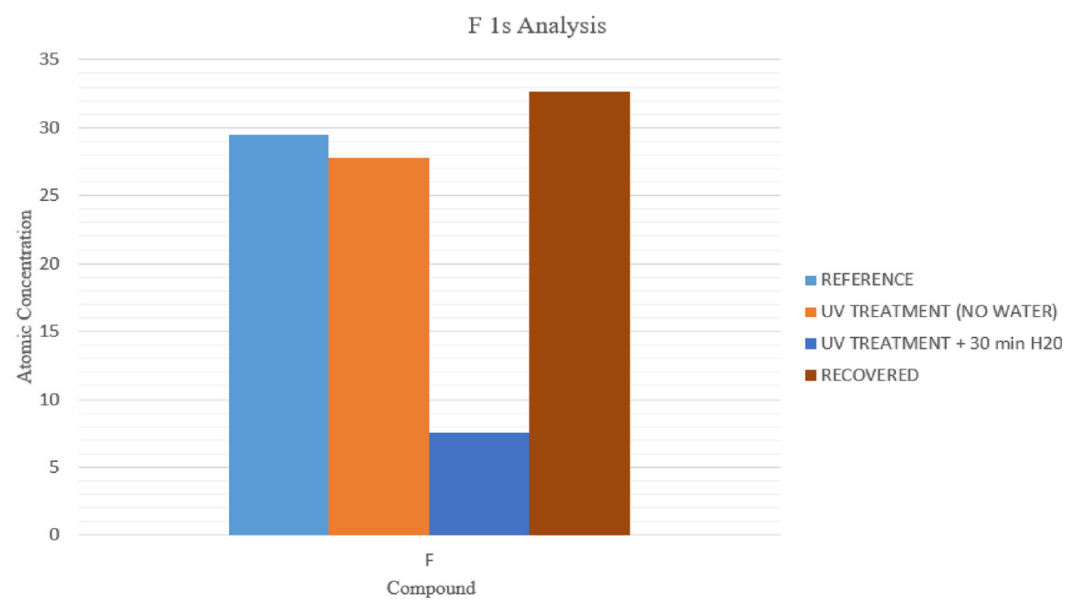

(c)

Fig. 7. Data Analysis of XPS spectrum: (a) Carbon analysis (b) Oxygen analysis (c) Fluor analysis.

\subsection{Chemical modification}

Four samples were tested by means of $\mathrm{x}$-ray spectroscopy measurements: The first sample was used as the reference, the second one was subjected to 6 min UV light treatment and the third one was exposed to $6 \mathrm{~min}$ UV light and after that $30 \mathrm{~min}$ under deionized water.
Finally, the fourth sample was exposed to the same UV treatment as the third one and then recovered by three-hour thermal treatment at $165^{\circ} \mathrm{C}$. In Fig. 6a-d the spectrums of each sample are shown. The spectrums show a clear difference in the sample treated with water, where the Fluor value has decreased while the oxygen content has dramatically increased. After the thermal recovery, these elements 


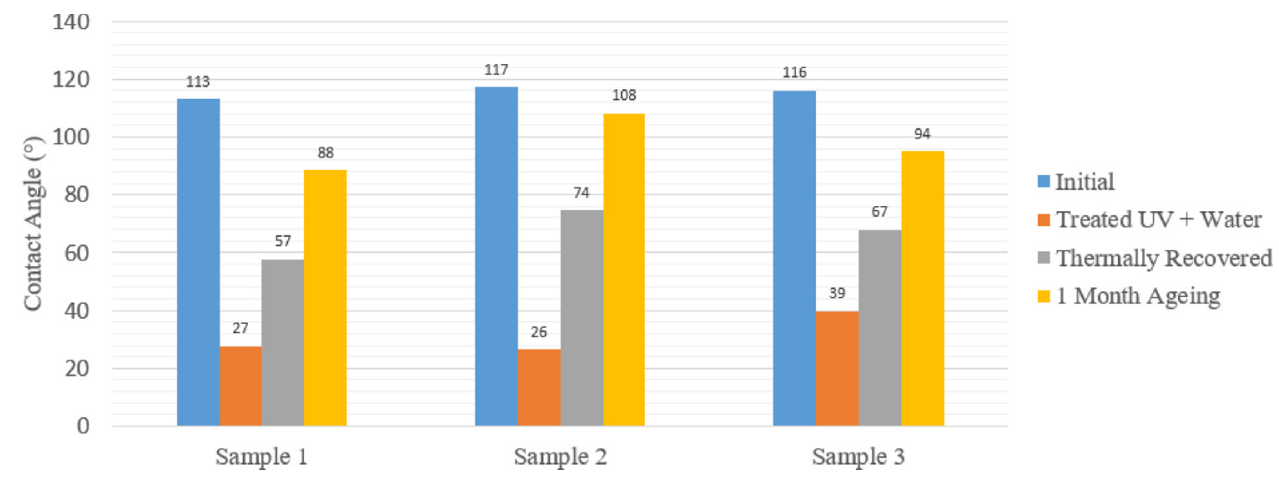

Fig. 8. Static contact angle measurements for 3 samples Aged during 1 Month after being treated and thermally recovered.

seem to return to values previous to the treatment. However, it is difficult to assess the real difference without computing the data in a different manner and excluding common groups attributed to carbon contamination. To analyse this, the concentration of each compound has been plotted in Fig. 7a-c.

In Fig. 7a it is easily observed that the groups identified as carbonates and -CF3 $(293.81 \mathrm{eV})$ follow a similar trend: The UV treatment slightly diminish its concentrations while in contrast, when the sample is submerged in deionized water, the concentration of both groups plummet. The thermal recovery returned these values to levels close to the reference. From Fig. $7 \mathrm{~b}$ it is clear that large quantities of oxygen are introduced in the sample when it is treated with water. In addition, Fig. 7c shows a process known as defluorination, where the Fluor content suffers a marked decrement. These changes have been reported as the main cause of surface modification from a chemical point of view $[7,19]$. As a consequence, the explanation for the change in hydrophobic behaviour could be similar to another already known process $[3,16]$. The exposition to UV light promotes cleaning and produce radical formation over the surface. This radical tends to link with other groups present during the treatment, mainly oxygen species. Nevertheless, these new bounds are not enough to change the surface behaviour. When the deionized water gets in contact with the treated surface a major chemical reaction takes place. During this reaction, further oxygen species are introduced on the surface. At the same time, water seems to facilitate the defluorination process.

In general, surface modification chemistry depends on oxygen species that link to radicals. Additionally, it depends on a slower process where the radicals connect each other producing interchain crosslinking [18]. This last process produces more stable surface properties since it creates a barrier that stops diffusion from the top into the bulk. The thermal hydrophobic recovery may be a consequence of three possible phenomena: reorientation of induced polar groups into the bulk, diffusion of species located on the surface and/or chains relaxation $[18,20]$ in the first monolayers at the surface. In this study, the surface recovery can be achieved easily by thermal treatment. This may indicate that crosslinking is not the dominant effect in the surface modification. Therefore, oxygen species that link to radicals could be the main responsible for this change.

In order to test if the recovery continues after the thermal treatment, 3 samples were fabricated, treated with UV exposition and water, thermally recovered and stored in standard room conditions at $25^{\circ} \mathrm{C}$ for one month. Fig. 8 shows that the recovery process slowly tends to the initial contact angle after the thermal treatment. In the three cases the recovered angle, i.e. the difference between the yellow and grey bars in Fig. 8 , is close to $30^{\circ}$, which may indicate that the recovery rate at standard conditions of pressure and temperature may be approximately constant. The recovery rate is defined by Eq. 2 .

Recovery Rate $=\frac{M A-T R}{T}$
Where $\mathrm{T}$ is the ageing time in days, MA is the angle measured after a period of $\mathrm{T}$ of ageing and TR is the contact angle of the sample thermally recovered.

Regarding the silicon content of the samples, it does not exceed $4 \%$ of the total composition. This species could be a remainder of the HMDS introduction during sample fabrication process steps and there is little possibility that it could modify the PTFE behaviour. Alumina concentration oscillates between 19 and $30 \%$ which can be related with the porosity of the PTFE film.

\section{Conclusions}

After analysing the data obtained from SEM, XPS, and static deionized water contact angle, we have shown that the adhesion of PTFE thin films can be perfectly controlled using non-coherent UV and water treatment. The exposure time, between 3 and $6 \mathrm{~min}$, is directly related to the contact angle of the deionized water over the sample and, consequently, with the increment of surface energy. The SEM images confirmed that the treatment does not produce any appreciable change in morphology. On the other hand, XPS analysis clearly shows that the surface modification is a consequence of defluorination and change in oxygen content. When the samples are submerged underwater, the concentration of oxygen dramatically increases from $15 \%$ to $35 \%$. Meanwhile, Fluor concentration decreased from $28 \%$ to $8 \%$. This phenomenon highly resembles the chemical changes that occur during oxygen plasma treatment and chemical etching. Since these late processes usually produce morphological changes that are not fully recoverable, our process can be a useful alternative for EWOD applications, where the modification should not be permanent. The original hydrophobic behaviour of the surface is recovered up to $98 \%$ after $3 \mathrm{~h}$ of thermal treatment at low temperatures. In addition, the process is cheap and easy to implement as it does not require a vacuum chamber and it can be performed under normal conditions of pressure and temperature.

\section{Intellectual property}

We confirm that we have given due consideration to the protection of intellectual property associated with this work and that there are no impediments to publication, including the timing of publication, with respect to intellectual property. In so doing we confirm that we have followed the regulations of our institutions concerning intellectual property.

\section{Research ethics}

Written consent to publish potentially identifying information, such as details or the case and photographs, was obtained from the patient(s) or their legal guardian(s). 


\section{Declaration of Competing Interest}

The authors declare that they have no known competing financial interests or personal relationships that could have appeared to influence the work reported in this paper.

\section{Acknowledgments}

This work has been partially supported by the Spanish Ministry of Science and Innovation under projects TEC2017-82305-R, RTI2018098728-B-C33. The activity was carried out under a program of, and funded by, the European Space Agency under project ESA AO/1-8876/ $17 / \mathrm{NL} / \mathrm{CRS}$

\section{References}

[1] J. Li, Z. Jing, F. Zha, Y. Yang, O. Wang, Z. Lei, Facile Spray-Coating Process for the Fabrication of Tunable Adhesive Superhydrophobic Surfaces with Heterogeneous Chemical Compositions Used for Selective Transportation of Microdroplets with Different Volumes, ACS Appl. Mater. Interfaces 6 (11) (2014) 8868-8877.

[2] Y. Long, Y. Shen, H. Tian, Y. Yang, H. Feng, J. Li, Superwettable Coprinus comatus coated membranes used toward the controllable separation of emulsified oil/water mixtures, J. Memb. Sci. 565 (2018) 85-94.

[3] S.R. Kim, Surface modification of poly (tetrafluoroethylene) film by chemical etching, plasma, and ion beam treatments, J. Appl. Polym. Sci. 77 (9) (2000) 1913-1920.

[4] M. Stamm, Polymer surfaces and interfaces, Polymer Surfaces and Interfaces: Characterization, Modification and Applications, Springer, Berlin Heidelberg, 2008, p. 1 ISBN 978-3-540-73864-0.

[5] Y. Ohkubo, K. Ishihara, M. Shibahara, A. Nagatani, K. Honda, K. Endo,

K. Yamamura, Drastic improvement in adhesion property of polytetra-

fluoroethylene (PTFE) via heat-assisted plasma treatment using a heater, Sci. Rep. 7 (1) (2017) 9476.

[6] I.I. Filatova, N.A. Savastenko, V.A. Lyushkevi, N.I. Chubrik, S.V. Goncharik,
V.M. Astreiko, Comparative study of the effect of RF and DBD plasma treatment on a photocatalytic activity of ZnO-Based catalysts, High Temp. Mater. Process. 19 (34) (2015) 221-229.

[7] Esrom, J.Y. Hilmar Zhang, Ulrich Kogelschatz, Photochemical modification and etching of PTFE with excimer VUV/UV radiation, in: K.L. Mittal, K.-W. Lee (Eds.), Polymer Surfaces and Interfaces: Characterization, Modification and Application, USP, Utrecht, 1997, pp. 27-35.

[8] T. Jun, X. Qunji, Surface modification of PTFE by 60Co $\gamma$-ray irradiation, J. Appl. Polym. Sci. 69 (3) (1998) 435-441.

[9] F.H. Winslow, Photooxidation of high polymers, Pure Appl. Chem. 49 (4) (1977) 495-502.

[10] B.G. Ranby, J.F. Rabek, Photodegradation, Photo-oxidation, and Photostabilization of Polymers, Wiley, New York, 1975.

[11] L.J. Matienzo, J.A. Zimmerman, F.D. Egitto, Surface modification of fluoropolymers with vacuum ultraviolet irradiation, J. Vac. Sci. Technol. A Vac. Surf. Films 12 (5) (1994) 2662-2671.

[12] J. Li, Z. Jing, Y. Yang, F. Zha, L. Yan, Z. Lei, Reversible low adhesive to high adhesive superhydrophobicity transition on $\mathrm{ZnO}$ nanoparticle surfaces, Appl. Surf. Sci. 289 (2014) 1-5.

[13] H. Yi, M. Jiang, D. Huang, G. Zeng, C. Lai, M. Li, X. Huo, Synergistic effect of artificial enzyme and $2 \mathrm{D}$ nano-structured $\mathrm{Bi}_{2} \mathrm{WO}_{6}$ for eco-friendly and efficient biomimetic photocatalysis, Appl. Catal. B 250 (2019) 52-62.

[14] Y. Yang, Z. Zeng, G. Zeng, D. Huang, R. Xiao, C. Zhang, C. Zhou, Ti, Appl. Catal. B 258 (2019) 117956.

[15] D.L. Flamm, O. Auciello, Plasma Deposition, Treatment, and Etching of Polymers: the Treatment and Etching of Polymers, Elsevier, 2012.

[16] M. Ahmadi, S. Bermejo, L. Castañer, EWOD using nonaqueous liquids, Microelectron. Eng. 139 (2015) 19-25.

[17] L. Ferry, G. Vigier, R. Alexander-Katz, C. Garapon, Interaction between UV radiation and filled polytetrafluoroethylene (PTFE). I. Degradation processes, J. Polym. Sci. Part B: Polym. Phys. 36 (12) (1998) 2057-2067.

[18] Nagia Dawood, Surface treatment of PTFE polymer using RF-Plasma, Australian J. Bas. Applied Sci. 171-175 (2016) (2016) 2309-8414.

[19] Z. Luo, Y. Wang, Q. Yang, Y. Luo, S. Tan, T. Chen, Z. Xie, Influence of engineering environment on wetting properties and long-term stability of a superhydrophobic polymer coating, J. Polym. Res. 21 (5) (2014) 447.

[20] C. Borcia, I.L. Punga, G. Borcia, Surface properties and hydrophobic recovery of polymers treated by atmospheric-pressure plasma, Appl. Surf. Sci. 317 (2014) 103-110. 